\title{
A Comparative Study of a New Class of Gastric Acid Suppressant Agent Named Vonoparazan versus Esomeprazole for the Eradication of Helicobacter pylori
}

\author{
Masahiro Tsujimae Hiroshi Yamashita Hiroki Hashimura Chise Kano \\ Keiko Shimoyama Atsushi Kanamori Kei Matsumoto Akio Koizumi \\ Kenji Momose Takaaki Eguchi Takumi Fukuchi Mikio Fujita Akihiko Okada
}

Department of Gastroenterology and Hepatology, Saiseikai Nakatsu Hospital, Osaka, Japan

\section{Key Words}

Vonoprazan · Esomeprazole · Helicobacter pylori .

Eradication - Gastric acid

\begin{abstract}
Background: Helicobacter pylori eradication rates have decreased worldwide. Gastric acid inhibition during treatment is important to eradicate these bacteria successfully. A new potassium-competitive acid blocker, vonoprazan (VPZ), has been shown to achieve high eradication rates in a previous randomized controlled trial. Objective: To determine the efficacy of VPZ for H. pylori eradication. Methods: A total of 874 patients were enrolled; 431 received esomeprazole (EPZ) and 443 received VPZ. First-line regimens contained clarithromycin (CAM) $200 \mathrm{mg}$ b.i.d., amoxicillin $750 \mathrm{mg}$ b.i.d., and either EPZ 20 mg b.i.d. or VPZ 20 mg b.i.d. for 7 days. Metronidazole $250 \mathrm{mg}$ b.i.d. replaced CAM in the secondline regimens. The eradication of $H$. pylori was assessed by ${ }^{13} \mathrm{C}$-urea breath tests $4-8$ weeks after each therapy. Results: The overall first-line eradication rate was $79.9 \%$ (341/427) with EPZ vs. $86.3 \%(377 / 439)$ with VPZ $(p=0.019)$. The second-line eradication rate was $83.3 \%(45 / 51)$ with EPZ vs. $91.1 \%(41 / 45)$ with VPZ $(p=0.900)$. Conclusion: VPZ was sig-
\end{abstract}

\section{KARGER}

E-Mail karger@karger.com www.karger.com/dig

(C) 2016 The Author(s)

Published by S. Karger AG, Basel

\section{Karger}

Open access

This article is licensed under the Creative Commons AttributionNonCommercial-NoDerivatives 4.0 International License (CC BYNC-ND) (http://www.karger.com/Services/OpenAccessLicense) Usage and distribution for commercial purposes as well as any distribution of modified material requires written permission. nificantly more effective than EPZ for first-line treatment. However, for second-line treatment, there was no significant difference between EPZ and VPZ.

(C) 2016 The Author(s)

Published by S. Karger AG, Basel

\section{Introduction}

Helicobacter pylori infection causes chronic gastritis, peptic ulcers, mucosa-associated lymphoid tissue lymphoma, and gastric cancer [1]. Therefore, $H$. pylori eradication is recommended for the prevention of these diseases. The success rate of the standard therapy, which uses a proton pump inhibitor (PPI) with amoxicillin (AMX) and clarithromycin (CAM), has decreased in many parts of the world. This decrease appears to be caused by an increase in the prevalence of CAM-resistant strains of $H$. pylori. Indeed, a study conducted between 2000 and 2013 in Japan [2] found that the overall resistance rate to CAM was $31.1 \%$. However, eradication failure is caused not only by bacterial resistance to antimicrobial agents but also by insufficient acid inhibition during treatment, which degrades and destabilizes the 
Table 1. Baseline characteristics of patients enrolled into each group

\begin{tabular}{lccc}
\hline & $\begin{array}{l}\mathrm{EPZ} \\
(n=431)\end{array}$ & $\begin{array}{l}\text { VPZ } \\
(n=443)\end{array}$ & $p$ value \\
\hline Age, years, mean $\pm \mathrm{SD}$ & $60.5 \pm 12.2$ & $61.6 \pm 12.3$ & 0.172 \\
Gender, male/female & $219 / 238$ & $220 / 189$ & 0.085 \\
BMI, kg/m 2 & $22.9 \pm 3.3$ & $22.9 \pm 3.9$ & 0.884 \\
Alcohol (yes/no) & $217 / 209$ & $222 / 215$ & 0.968 \\
Smoking (yes/no) & $57 / 369$ & $84 / 350$ & 0.018 \\
\hline
\end{tabular}

BMI, body mass index; EPZ, esomeprazole; VPZ, vonoprazan.

antibiotics in the stomach [3]. Gastric acid inhibition by PPIs is influenced by the cytochrome P450 (CYP) 2C19 genotype and gastric emptying.

Vonoprazan (VPZ) is a novel oral potassium-competitive acid blocker that is part of a new class of gastric acidsuppressant agents. It has been available since February 2015 in Japan but is presently not yet approved in other countries. It competitively inhibits the binding of potassium ions to $\mathrm{H}^{+}, \mathrm{K}^{+}$-ATPase in the final step of acid secretion in gastric parietal cells. It has a potent and long-lasting anti-secretory effect on $\mathrm{H}^{+}, \mathrm{K}^{+}$-ATPase, owing to its high accumulation in and slow clearance from gastric tissues [4]. Several reports showed that the acid-inhibitory effects of VPZ were stronger than those of conventional PPIs $[5,6]$. A phase III randomized trial in Japan revealed that a VPZ regimen was more effective as part of a firstand second-line triple therapy than a lansoprazole (LPZ) regimen (92.6\%, VPZ regimen; 75.9\%, LPZ regimen) [7]. However, there are only a few reports in the literature about the $H$. pylori eradication rate of $\mathrm{VPZ}$ in a clinical setting. In addition, no comparative studies have been conducted related to esomeprazole (EPZ) and VPZ regimens. EPZ has been available for 5 years in Japan, and is considered to be less affected by CYP2C19 than other PPIs. The aim of this study was to compare the $H$. pylori eradication rate of EPZ and VPZ regimens.

\section{Methods}

\section{Patients}

A retrospective, open-label, single-center study design was adopted at Saiseikai Nakatsu Hospital, Osaka, Japan. A total of 874 patients who were diagnosed with $H$. pylori infection between November 2013 and June 2016 enrolled in the study. Four hundred thirty-one patients received the EPZ regimen from November 2013 to March 2015, while 443 patients received the VPZ regimen from April 2015 to June 2016. Before treatment, demographical and clinical characteristics including age, body mass index (BMI), smoking status, and alcohol consumption were checked. Most patients had also undergone an upper gastrointestinal endoscopy before enrolment. All patients in our hospital received an endoscopy before eradication as a screening process against gastric cancer. However, a few patients who were diagnosed at other hospitals did not. We have excluded patients with a history of eradication and gastric operations from this study. The protocol and informed consent forms were reviewed and approved by the Ethical Committee of Saiseikai Nakatsu Hospital before the start of the study. This study was conducted in accordance with the Declaration of Helsinki and the consolidated Good Clinical Practice guidelines.

Assessment of H. pylori Infection and Eradication Therapy

The presence of $H$. pylori infection was detected by ${ }^{13} \mathrm{C}$-urea breath tests, serological testing (HM-CAP kit, Enteric Product Inc., Westbury, NY, USA), and the rapid urease test (Helico Check, Otsuka Co., Tokushima, Japan).

First-line eradication regimens consisted of CAM $200 \mathrm{mg}$ b.i.d., AMX 750 mg b.i.d., and either EPZ 20 mg b.i.d. or VPZ 20 mg b.i.d., for 7 days. Patients were instructed to take the triple therapy once in the morning and once in the evening. At least $4-8$ weeks after the therapy, the extent of eradication of $H$. pylori infection was assessed by a ${ }^{13} \mathrm{C}$-urea breath test. When eradication failed, that is, if the bacteria were still present, the patients underwent second-line eradication treatment. The second-line eradication regimens consisted of metronidazole (MNZ) $250 \mathrm{mg}$ b.i.d., AMX $750 \mathrm{mg}$ b.i.d., and either EPZ $20 \mathrm{mg}$ b.i.d. or VPZ $20 \mathrm{mg}$ b.i.d., for 7 days. At least 4-8 weeks after the second-line therapy, the extent of eradication of $H$. pylori infection was again assessed by a ${ }^{13} \mathrm{C}$-urea breath test. Successful eradication of $H$. pylori was defined as a result when the extent of bacterial presence was less than $2.5 \%$. All patients were interviewed by a doctor to document adverse events (if any) experienced and to determine the drug compliance.

\section{Data Analysis}

The cure rate was defined as the number of successfully treated patients divided by the number of treated patients. The cure rate was evaluated in 2 ways: intention-to-treat (ITT), which included all eligible patients enrolled in the study, regardless of compliance; and per-protocol (PP), which excluded patients whose compliance was poor or patients with unavailable data. Demographic characteristics were analyzed by the $t$ test. Comparison of categorized data was analyzed by the $\chi^{2}$ test. All $p$ values were 2 -sided, and $p<$ 0.05 was selected to indicate statistical significance. The primary endpoint was comparison of the first-line eradication rate between the EPZ and VPZ regimens in the ITT and PP groups, while the secondary endpoint was comparison of the second-line eradication rate between the 2 regimens in the ITT and PP groups. This study aimed to reveal the differences in the eradication rate with respect to the patient's background.

\section{Results}

\section{Demographic Characteristics of Patients}

Eight hundred and seventy-four subjects were included in the trial (431 patients in the EPZ group; 443 patients in the VPZ group). The baseline characteristics of each 


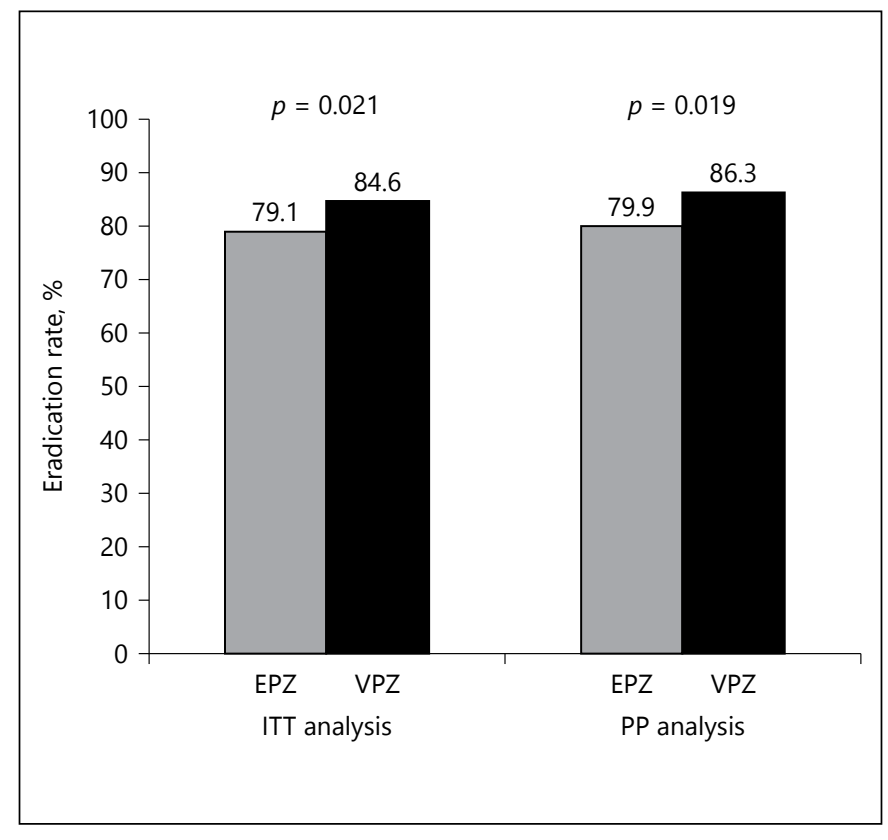

Fig. 1. H. pylori eradication rates in first-line triple therapy. Both ITT and PP analyses showed that the VPZ regimen was superior to the EPZ regimen. VPZ, vonoprazan; EPZ, esomeprazole; ITT, intention to treat; $\mathrm{PP}$, per protocol.

group are summarized in Table 1 . There was no significant difference between the 2 treatment groups except for smoking history. Both treatment groups achieved $>90 \%$ drug compliance. The overall first-line eradication rates combining both the VPZ and EPZ regimens were $82.2 \%$ $(718 / 874)$ by ITT analysis and $82.9 \%(718 / 866)$ by PP analysis. In contrast, the combined second-line eradication rates were $86.0 \%(86 / 100)$ by ITT analysis and $90.0 \%$ $(86 / 96)$ by $\mathrm{PP}$ analysis.

\section{Comparison of Eradication Rate}

The overall first-line eradication rate was $79.1 \%$ (341/431) for the EPZ regimen and 84.6\% (377/443) for the VPZ regimen by ITT analysis; the eradication rate by PP analysis for EPZ and VPZ regimens was 79.9\% (341/427) and $86.3 \%$ (377/439) respectively. The eradication rate for VPZ regimen was significantly higher than that for EPZ regimen in both ITT $(p=0.021)$ and $\mathrm{PP}(p=$ 0.019) analyses (Fig. 1).

The overall second-line eradication rate was $83.3 \%$ (45/54) and $89.1 \%(41 / 46)$ for EPZ and VPZ regimens, respectively, by ITT analysis. In PP analysis, the eradication rate was $88.2 \%(45 / 51)$ for the EPZ regimen and 91.1\% (41/45) for the VPZ regimen (Fig. 2). A statistically significant difference in eradication rate was not

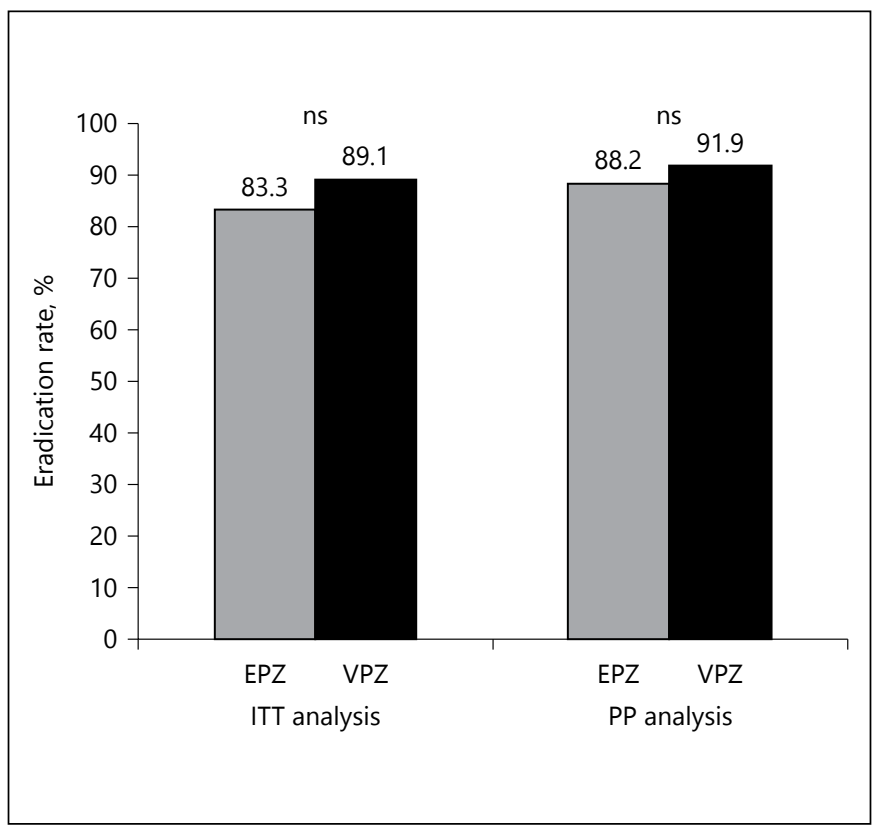

Fig. 2. H. pylori eradication rates in second-line triple therapy. Statistical significance was not found between the VPZ and EPZ regimens. VPZ, vonoprazan; EPZ, esomeprazole; ITT, intention to treat; PP, per protocol; ns, not significant.

found between the 2 groups both in ITT ( $p=0.587)$ and PP $(p=0.900)$ analyses.

In terms of background disease, the eradication rate of the VPZ regimen was significantly higher than that of the EPZ regimen in patients with antral-predominant gastritis. For other background diseases, there were no significant differences between the 2 groups (Table 2). In particular, the eradication rate of the VPZ regimen was higher than that of the EPZ regimen in diseases in which acid secretion persists, such as duodenal ulcers and antral-predominant gastritis.

When comparing patient-specific eradication rates between the regimens, a significant difference was seen in patients who were less than 70 years of age, female, or had a BMI $<25$ (Table 3). The eradication rate of the VPZ regimen was significantly higher than that of the EPZ regimen (age $<70: 85.4$ vs. $78.5 \%, p=0.024$; female: 86.4 vs. $76.2 \%, p=0.005$; BMI <25: 85.5 vs. $79.5 \%, p=0.041$ ). There were no significant differences in eradication rates between the 2 groups with respect to other factors.

\section{Adverse Events}

Both VPZ and EPZ regimens were well tolerated by the patients. No severe adverse effects were reported; however, some patients experienced less severe adverse effects including diarrhea, bitter taste, nausea, and rash (Table 4). 
Table 2. Comparison of eradication rate background disease

\begin{tabular}{lllll}
\hline & EPZ & & VPZ & $p$ value \\
\cline { 2 - 2 } & eradication rate $(n)$ & & eradication rate $(n)$ & \\
\hline Gastritis (antral-predominant) & $77.5(134 / 173)$ & & $86.5(141 / 163)$ & 0.032 \\
Gastritis (pangastilitis) & $81.5(207 / 254)$ & & $85.8(218 / 254)$ & 0.187 \\
Gastric ulcer & $88.9(32 / 36)$ & $87.5(49 / 56)$ & 0.898 \\
Duodenal ulcer & $78.9(15 / 19)$ & $93(40 / 43)$ & 0.374 \\
Gastric cancer & $89.5(17 / 19)$ & $75(21 / 28)$ & 0.390 \\
\hline
\end{tabular}

EPZ, esomeprazole; VPZ, vonoprazan.

Table 3. Comparison of patient-specific eradication rate

\begin{tabular}{|c|c|c|c|}
\hline & EPZ & VPZ & $p$ value \\
\hline & eradication rate $(n)$ & eradication rate $(n)$ & \\
\hline \multicolumn{4}{|l|}{ Age, years } \\
\hline$>70$ & $84.0(89 / 106)$ & $87.1(108 / 124)$ & 0.499 \\
\hline$<70$ & $78.5(252 / 321)$ & $85.4(269 / 315)$ & 0.024 \\
\hline \multicolumn{4}{|l|}{ Gender } \\
\hline Male & $82.8(197 / 238)$ & $85.4(187 / 219)$ & 0.446 \\
\hline Female & $76.2(144 / 189)$ & $86.4(190 / 220)$ & 0.008 \\
\hline \multicolumn{4}{|l|}{ BMI } \\
\hline$>25$ & $82.0(73 / 89)$ & $86.4(89 / 103)$ & 0.404 \\
\hline$<25$ & $79.5(263 / 331)$ & $85.5(283 / 331)$ & 0.041 \\
\hline \multicolumn{4}{|l|}{ Alcohol } \\
\hline Yes & $79.6(176 / 221)$ & $85.8(188 / 219)$ & 0.085 \\
\hline No & $80.0(164 / 205)$ & $85.6(184 / 215)$ & 0.129 \\
\hline \multicolumn{4}{|l|}{ Smoking } \\
\hline Yes & $77.2(44 / 57)$ & $88.1(74 / 84)$ & 0.086 \\
\hline No & $80.2(296 / 369)$ & $85.1(298 / 350)$ & 0.081 \\
\hline
\end{tabular}

BMI, body mass index; EPZ, esomeprazole; VPZ, vonoprazan.

A total of 8 patients deviated from the protocol, out of which 4 (one receiving VPZ, 3 receiving EPZ) did not complete the protocol due to adverse effects.

\section{Discussion}

This study showed that the first-line $H$. pylori eradication rate of the VPZ regimen was significantly higher than that of the EPZ regimen in both ITT and PP analyses.

Both CAM and AMX are acid-sensitive; therefore, gastric acid secretions must be strongly inhibited to prevent degradation of the drug during eradication therapy. Sufficient inhibition of gastric acid to generate a stomach
$\mathrm{pH}>5$ increases the stability and bioavailability of these acid-sensitive antibiotics $[8,9]$.

Hunt and Scarpignato [10] suggested a reason for the observed superiority of VPZ over conventional PPIs by highlighting the differences between VPZ and conventional PPIs for night-time control of intragastric acidity. Long-lasting acid suppression is important for eradication success. Patients receiving conventional PPIs may experience inadequate acid-inhibition during the night (commonly known as nocturnal acid breakthrough), which may interfere with the stability and bioavailability of acid-sensitive antibiotics and thereby decrease the eradication rate. Sakurai et al. [7] compared the $24 \mathrm{~h}$ mean gastric $\mathrm{pH}$ on day one between VPZ $20 \mathrm{mg}$ and $\mathrm{EPZ}$ 
Table 4. Breakdown of adverse effects

\begin{tabular}{|c|c|c|c|c|}
\hline & \multicolumn{2}{|c|}{$\begin{array}{l}\text { EPZ regimen } \\
(n=427)\end{array}$} & \multicolumn{2}{|c|}{$\begin{array}{l}\text { VPZ regimen } \\
(n=439)\end{array}$} \\
\hline & $\%$ & $n$ & $\%$ & $n$ \\
\hline Total & 1.17 & 5 & 0.68 & 3 \\
\hline Diarrhea & 0.47 & 2 & 0.23 & 1 \\
\hline Rash & 0.23 & 1 & 0.23 & 1 \\
\hline Nausea & 0.23 & 1 & 0.23 & 1 \\
\hline Bitter taste & 0.23 & 1 & & \\
\hline
\end{tabular}

$20 \mathrm{mg}$, and reported that the intragastric $\mathrm{pH}$ after $\mathrm{VPZ}$ treatment ( $\mathrm{pH}$ 5.2) was significantly higher than that after EPZ treatment ( $\mathrm{pH} 3.0)$. Hence, the rapid and sustained acid-inhibitory effect of $\mathrm{VPZ}$ might be responsible for its superior eradication rate compared with that of EPZ.

A previous phase III randomized trial in Japan showed a high eradication rate of $92 \%$ for the VPZ regimen [7]. The patients enrolled in that study had only gastric or duodenal ulcers. When we analyzed only those patients with a history of ulcers, the rate of $H$. pylori eradication was $89.9 \%(89 / 99)$, which was similar to that of the aforementioned trial. In addition, Shinozaki et al. [11] evaluated $H$. pylori eradication rate in patients with gastritis and ulcers and reported that the eradication rate of the VPZ regimen was $85 \%$. Collectively, these data indicate that the eradication rate following VPZ regimen in a clinical setting can be expected to reach 85-90\%.

Patients with mild stomach atrophy showed higher eradication rates when using the VPZ regimen. A recent study reported that severe atrophy decreased the success rate of eradication [12]. Hence, it is recommended that $H$. pylori be eradicated before it causes severe stomach gastritis. A significant difference in eradication rate was seen in patients who were younger than 70 years or who had BMI $<25$ between the 2 groups in this study. The VPZ regimen had a competitive advantage over the EPZ regimen in these patients. A previous study showed that young, healthy individuals had high mean basal and maximal acid outputs, which correlated negatively with body weight [13]. It is assumed that patients with lower BMI had higher levels of acid secretion. It is conceivable that younger patients also had higher acid secretion levels than older patients because the atrophy of the stomach progressed gradually in $H$. pylori-positive patients with an increase in age. Therefore, we assumed that this is the reason for the higher eradication rate of the VPZ regimen in these patient groups. Since VPZ has a more potent acid-inhibitory effect than conventional PPIs, the VPZ regimen was superior in these patient groups. Interestingly, Ishimura et al. [14] showed that maximal acid output decreased gradually with age in male patients but remained unchanged in female patients, in healthy Japanese subjects over the past 2 decades. This may be a reason for the higher eradication rate following VPZ regimen in female patients. Furthermore, some studies revealed that women showed lower $H$. pylori eradication rates when receiving a PPI, MNZ, and AMX triple therapy $[15,16]$. Hence, the VPZ regimen may be a more effective therapy for resolving these problems.

In this study, there were no significant differences in patient background except for smoking status. Kamada et al. [17] showed that smoking exerted a negative influence on the eradication rate. The group that received the VPZ regimen had a higher number of smokers. Nevertheless, the eradication rate in the VPZ regimen group was higher than that in the EPZ regimen group. This result is probably due to the superiority of the VPZ regimen. Ishioka et al. [18] reported that treatment with CAM $800 \mathrm{mg}$ b.i.d resulted in a higher eradication rate in triple therapy using conventional PPIs. Further study is necessary to conclude whether or not patients with smoking status should also be administered CAM $800 \mathrm{mg}$ b.i.d in a VPZ-based triple therapy.

In contrast to the first-line therapy, there was no difference in the eradication rate following second-line therapy. One reason for this is the low resistance rate to $\mathrm{MNZ}$ (2-5\%) for H. pylori in Japan [19]. Thus, acid suppression should not be a major problem that affects eradication rate. The prevalence of antibiotic resistance to CAM, $\mathrm{MNZ}$, and levofloxacin appears to be rapidly increasing worldwide [20]. We are currently facing a dilemma between risking an increase in drug tolerance and a decreasing eradication rate. It is important to use antibiotics appropriately but changing to a VPZ-based treatment may offer a solution to this situation.

The strength of this study is that the efficacy of the VPZ regimen for $H$. pylori eradication was evaluated in a larger cohort than in the previous phase III study. However, the limitations of this study are as follows: the study followed an open-labeled, retrospective, single-center design. We also could not evaluate the effect of CYP2C19 genotype and antibiotic resistance in this study. However, we believe the change in tolerance to antibiotics is relatively small, since this study was performed in the same hospital for a period of 3 years. 
The antibiotic doses were lower and the treatment duration shorter in the present study than in the Toronto Consensus [21]. The choice of CAM $200 \mathrm{mg}$ b.i.d. is not consistent with guidelines in Western countries. Moreover, the AMX dose is also less than that typically used in the West. We expected that the total amount of antimicrobial drugs can be reduced because VPZ exerts a powerful acid inhibitory effect. In Japan, the use of CAM $400 \mathrm{mg}$ b.i.d. and AMX 1,000 mg b.i.d. for a treatment duration of 14 days is not widely practiced. One reason for this lies in the Japanese health insurance design. For example, the maximum amount of AMX is limited to $1,500 \mathrm{mg}$ per day for all infectious diseases. Since VPZ strongly inhibits acid secretion, a satisfactory eradication rate was achieved with the minimum amount of antibacterial drugs. A sub-analysis of the previous VPZ randomized controlled trial [7] showed that there was no significant difference in the eradication rate between the CAM 200 and $400 \mathrm{mg}$ b.i.d. regimens when using VPZ. Shortening the treatment period and reducing the amount of antimicrobial agent may result in a decrease in side effects. Furthermore, the compliance rate will improve and medical expenses can be reduced. In our study, the total first-line eradication rate was $82.2 \%(718 / 874)$ for both VPZ and EPZ regimens together, which was a significant result. We did not investigate whether a longer therapy duration and greater amount of antimicrobial agent contributed to higher eradication rates, especially with regard to the VPZ regimen. Further studies are required before adapting Western consensus.

\section{Conclusions}

The first-line H. pylori eradication rate of the VPZ regimen was significantly higher than that of the EPZ regimen for all patients, irrespective of their background. VPZ could be a useful alternative to PPIs, in combination with antibiotics for the eradication of $H$. pylori.

\section{Funding}

This research received no specific grant from any funding agency in the public, commercial, or non-profit sectors.

\section{Acknowledgments}

The authors thank medical billing clerks for their help in data collection.

\section{Statement of Ethics}

We followed ethical guidelines such as the Helsinki Declaration, ethical guidelines on medical research for people in Japan, and guidelines for the proper handling of personal information in the medical field. We also obtained approval from the ethics committee of our facility.

\section{Disclosure Statement}

The authors declare no conflicts of interest.

\section{References}

1 Murakami K, Sakurai Y, Shiino M, Funao N, Nishimura A, Asaka M: Vonoprazan, a novel potassium-competitive acid blocker, as a component of first-line and second-line triple therapy for Helicobacter pylori eradication: a phase III, randomised, double-blind study. Gut 2016;65:1439-1446.

2 McColl KE: Clinical practice. Helicobacter pylori infection. N Engl J Med 2010;362:15971604.

3 Okamura T, Suga T, Nagaya T, Arakura N, Matsumoto T, Nakayama Y, Tanaka E: Antimicrobial resistance and characteristics of eradication therapy of Helicobacter pylori in Japan: a multi-generational comparison. Helicobacter 2014;19:214-220.

4 Hunt $\mathrm{RH}: \mathrm{pH}$ and $\mathrm{Hp}$ - gastric acid secretion and Helicobacter pylori: implications for ulcer healing and eradication of the organism. Am J Gastroenterol 1993;88:481483.
5 Shin JM, Inatomi N, Munson K, Strugatsky D, Tokhtaeva E, Vagin O, Sachs G: Characterization of a novel potassium-competitive acid blocker of the gastric H,K-ATPase, 1 - [5 - (2 - fluorophenyl) - 1 - (pyridin3-ylsulfonyl)-1H-pyrrol-3-yl]-N-methylmethanamine monofumarate (TAK-438). J Pharmacol Exp Ther 2011;339:412-420.

6 Kagami T, Sahara S, Ichikawa H, Uotani T, Yamade $M$, Sugimoto $M$, Hamaya $Y$, Iwaizumi M, Osawa S, Sugimoto K, Miyajima H, Furuta T: Potent acid inhibition by vonoprazan in comparison with esomeprazole, with reference to CYP2C19 genotype. Aliment Pharmacol Ther 2016;43:10481059.

7 Sakurai Y, Mori Y, Okamoto H, Nishimura A, Komura E, Araki T, Shiramoto M: Acidinhibitory effects of vonoprazan $20 \mathrm{mg}$ compared with esomeprazole $20 \mathrm{mg}$ or rabeprazole $10 \mathrm{mg}$ in healthy adult male sub- jects - a randomised open-label cross-over study. Aliment Pharmacol Ther 2015;42: 719-730.

8 Grayson ML, Eliopoulos GM, Ferraro MJ, Moellering RC Jr: Effect of varying $\mathrm{pH}$ on the susceptibility of Campylobacter pylori to antimicrobial agents. Eur J Clin Microbiol Infect Dis 1989;8:888-889.

9 Sachs G, Meyer-Rosberg K, Scott DR, Melchers K: Acid, protons and Helicobacter pylori. Yale J Biol Med 1996;69:301-316.

10 Hunt RH, Scarpignato C: Potassium-competitive acid blockers ( $\mathrm{P}-\mathrm{CABs})$ : are they finally ready for prime time in acid-related disease? Clin Transl Gastroenterol 2015;6:e119.

11 Shinozaki S, Nomoto H, Kondo Y, Sakamoto $\mathrm{H}$, Hayashi Y, Yamamoto H, Lefor AK, Osawa $\mathrm{H}$ : Comparison of vonoprazan and proton pump inhibitors for eradication of Helicobacter pylori. Kaohsiung J Med Sci 2016;32: 255-260. 
12 Kalkan IH, Sapmaz F, Guliter S, Atasoy P: Severe gastritis decreases success rate of Helicobacter pylori eradication. Wien Klin Wochenschr 2016;128:329-334.

13 Novis BH, Marks IN, Bank S, Sloan AW: The relation between gastric acid secretion and body habitus, blood groups, smoking, and the subsequent development of dyspepsia and duodenal ulcer. Gut 1973;14:107-112.

14 Ishimura N, Owada Y, Aimi M, Oshima T, Kamada T, Inoue K, Mikami H, Takeuchi T, Miwa H, Higuchi K, Kinoshita Y: No increase in gastric acid secretion in healthy Japanese over the past two decades. J Gastroenterol 2015;50:844-852.
15 Osato MS, Reddy R, Reddy SG, Penland RL, Malaty HM, Graham DY: Pattern of primary resistance of Helicobacter pylori to metronidazole or clarithromycin in the United States. Arch Intern Med 2001;161:1217-1220.

16 Cai W, Zhou L, Ren W, Deng L, Yu M: Variables influencing outcome of Helicobacter pylori eradication therapy in South China. Helicobacter 2009;14:91-96.

17 Kamada T, Haruma K, Komoto K, Mihara M, Chen X, Yoshihara M, Sumii K, Kajiyama G, Tahara K, Kawamura Y: Effect of smoking and histological gastritis severity on the rate of $H$. pylori eradication with omeprazole, amoxicillin, and clarithromycin. Helicobacter 1999;4:204-210.

18 Ishioka H, Mizuno M, Take S, Ishiki K, Nagahara Y, Yoshida T, Okada H, Yokota K, Oguma K: A better cure rate with $800 \mathrm{mg}$ than with $400 \mathrm{mg}$ clarithromycin regimens in oneweek triple therapy for Helicobacter pylori infection in cigarette-smoking peptic ulcer patients. Digestion 2007;75:63-68.
19 Kato M, Yamaoka Y, Kim JJ, Reddy R, Asaka M, Kashima K, Osato MS, El-Zaatari FA, Graham DY, Kwon DH: Regional differences in metronidazole resistance and increasing clarithromycin resistance among Helicobacter pylori isolates from Japan. Antimicrob Agents Chemother 2000;44:2214-2216.

20 Thung I, Aramin H, Vavinskaya V, Gupta S, Park JY, Crowe SE, Valasek MA: Review article: the global emergence of Helicobacter pylori antibiotic resistance. Aliment Pharmacol Ther 2016;43:514-533.

21 Fallone CA, Chiba N, van Zanten SV, Fischbach L, Gisbert JP, Hunt RH, Jones NL, Render C, Leontiadis GI, Moayyedi P, Marshall JK: The Toronto Consensus for the treatment of Helicobacter pylori infection in adults. Gastroenterology 2016;151:51-69.e14. 